\title{
A Neonatal Form of Alexander Disease Presented with Intractable Seizures and Obstructive Hydrocephalus
}

\author{
Il Han Yoo', Won Gi Hong, Hunmin Kim², Byung Chan Lim", Hee Hwang' ${ }^{1}$, Jong-Hee Chae ${ }^{1,3}$, Ki Joong Kim, ${ }^{1,2}$ and Yong Seung Hwang ${ }^{1,2}$ \\ 'Pediatric Clinical Neuroscience Center, Department of Pediatrics, Seoul National University Children's hospital, Seoul, Korea \\ ${ }^{2}$ Department of Pediatrics, Seoul National University Bundang Hospital, Gyeonggi-do, ${ }^{3}$ Department of Pediatrics, Seoul National University College \\ of Medicine, Seoul, Korea
}

\begin{abstract}
Alexander disease is a rare degenerative leukodystrophy caused by dominant mutations in glial fibrillary acidic protein (GFAP). The neonatal form of Alexander disease may manifest as frequent and intractable seizures or obstructive hydrocephalus, with rapid progression leading to severe disability or death within two years. We report a case of a 50-day-old male who presented with intractable seizures and obstructive hydrocephalus. His initial magnetic resonance imaging (MRI) suggested a tumor-like lesion in the tectal area causing obstructive hydrocephalus. Despite endoscopic third ventriculostomy and multiple administrations of antiepileptic drugs, the patient experienced intractable seizures with rapid deterioration of his clinical status. After reviewing serial brain MRI scans, Alexander disease was suspected. Subsequently, we confirmed the de novo missense mutation in GFAP (c.1096T>C, Y366H). Although the onset was slightly delayed from the neonatal period (50 days old), we concluded that the overall clinical features were consistent with the neonatal form of Alexander disease. Furthermore, we also suspected that a Y366 residue might be closely linked to the neonatal form of Alexander disease based on a literature review.
\end{abstract}

Key words: Alexander disease, GFAP mutation, Hydrocephalus, Seizure

\section{Introduction}

Alexander disease, first described by W. Stewart Alexander in $1949,{ }^{1)}$ is a rare neurodegenerative disorder caused by dominant mutations in the glial fibrillary acidic protein (GFAP) gene. These mutations produce a gain of function in a GFAP protein leading to astrocyte dysfunction, deposition of Rosenthal fibers, and abnormal intracytoplasmic inclusions in astrocytes. Although this rare leukoencephalopathy has been well known for its frequent associations with macrocephaly or megalencephaly, the clinical presentation is rather non-specific, including seizures, hydrocephalus, megalencephaly, progressive psychomotor retardation, and bulbar/pseudobulbar signs. The recent study showed that these clinical symptoms and signs may vary according to age at onset and GFAP mutation site. ${ }^{2)}$

This study by Prust et al. includes a review of 218 cases, including 30 new cases of Alexander disease, with evidence suggesting that early age of onset (often before 4 years) is frequently associated with seizures, macrocephaly, encephalopathy, paroxysmal deterioration, or failure to thrive. However, only a few studies emphasizes in distinguishing a neonatal form of Alexander disease from early onset type where specific clinical symptoms exhibit during neonatal period. ${ }^{3,4)}$ The neonatal form can be distinguished from others by frequent intractable seizures in

\footnotetext{
Received: 23 August 2013, Revised: 23 September 2013, Accepted: 26 September 2013, Published: 31 December 2013

*Corresponding author: Byung Chan Lim, M.D.

Department of Pediatrics, Seoul National University College of Medicine, Seoul National University Hospital, 101 Daehak-ro, Jongno-gu, Seoul 110-744, Korea

Tel: +82-2-2072-2364, Fax: +82-2-743 3455, E-mail: prabbit7@snu.ac.kr

${ }^{*}$ Conflict of interest: We declare that we do not have any conflicts of interests.

(c) This is an open-access article distributed under the terms of the Creative Commons Attribution Non-Commercial License (http://creativecommons.org/licenses/by-nc/3.0/) which permits unrestricted non-commercial use, distribution, and reproduction in any medium, provided the original work is properly cited.

(c) Copyright 2013 by the Korean Society of Medical Genetics www.e-kjgm.org
} 
addition to the presence of leukodystrophy. Ataxia, hyperreflexia, and other upper motor neuron signs are usually absent in the neonatal form. Furthermore, it may be misdiagnosed as a neoplasm, since enhancing brain stem lesions are also associated with obstructive hydrocephalus.

To confirm the diagnosis of Alexander disease, GFAPsequencing is more widely used than immunohistochemical staining of brain biopsy material. Accordingly, some genotypephenotype correlations have been reported; for example, mutations at residue $\mathrm{R} 79$ and $\mathrm{R} 239$ were more prevalent in the early onset group. ${ }^{2,5}$ However, there has been limited genotype data regarding the neonatal form of Alexander disease.

In the present study, we report a case of Alexander disease, which could be classified as the neonatal form based on the characteristic clinical features including onset age (50 days), intractable seizures, and obstructive hydrocephalus caused by tumor-like lesion in the tectal area. We also confirmed this diagnosis through genetic testing of GFAP.

\section{Case report}

A boy was born at 38 weeks and 5 days gestation via vaginal delivery to non-consanguineous Korean parents. His birth weight was $2.9 \mathrm{~kg}$ (10-25 percentile) with no reported head circumference. At the age of 50 days, he had clonic movements in his arms and face, which lasted for about one minute. Initially, this event occurred one or two times per day. However, the frequency of these events gradually increased to up to 10 times per day. He was admitted for evaluation and management of these repeated seizures. Upon admission, head circumference, height, and weight were $40.5 \mathrm{~cm}$ (90-95 percentile), $60.3 \mathrm{~cm}$ (75-90 percentile), and $5.3 \mathrm{~kg}$ (10-25 percentile), respectively. Muscle tone and deep tendon reflexes were slightly decreased symmetrically. Babinski reflexes were present on both sides. Electroencephalography revealed focal epileptiform discharges from the left frontal area. Brain magnetic resonance imaging (MRI) revealed dilatation of both lateral ventricles and the third ventricle. Abnormally high T2 weighted signal intensity was noted in the periventricular white matter, basal ganglia, and midbrain including the tectum. The tectal lesion also showed focal enhancement with enlargement (Fig. 1A and B). Initially, the suspected diagnosis was a congenital infection such as cytomegalovirus or tectal glioma causing obstructive hydrocephalus. Endoscopic third ventriculostomy was performed as management for hydrocephalus. Although ventricular dilatation improved in subsequent brain MRIs, the extent of periventricular white matter, basal ganglia, and midbrain lesions increased with rapidly progressive cortical atrophy (Fig. 1C-F). Seizures were refractory to multiple antiepileptic drugs.

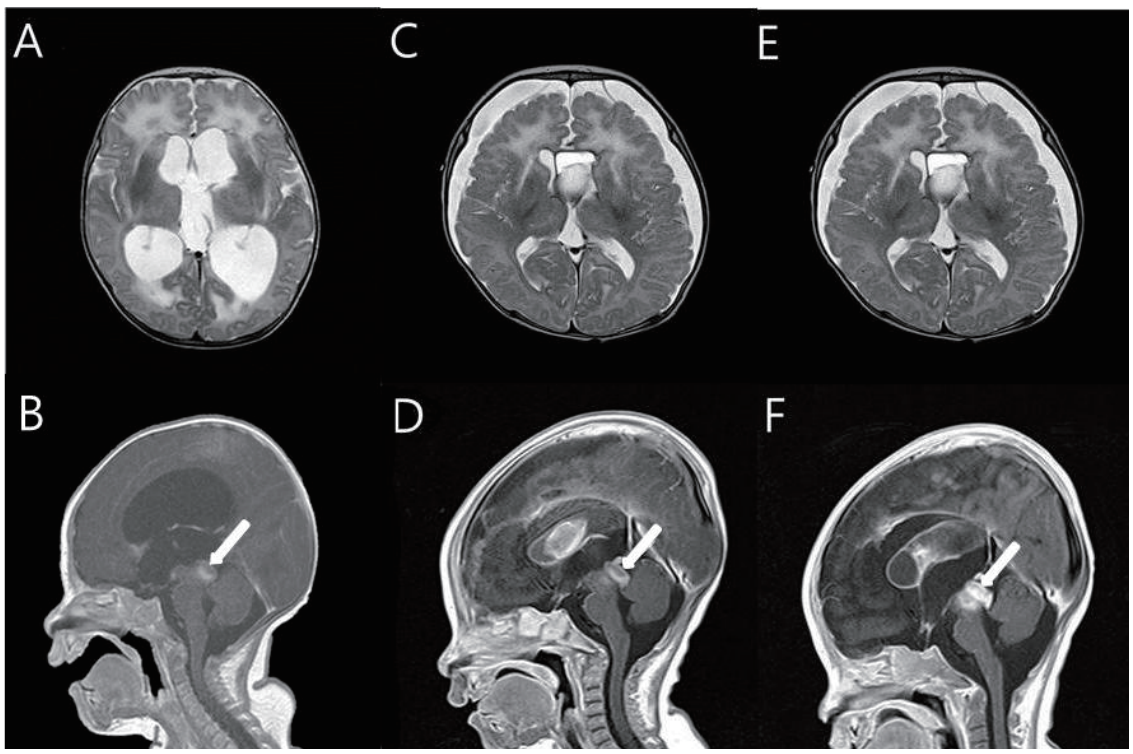

Fig. 1. Serial T2 weighted axial and contrast enhanced sagittal brain magnetic resonance image findings. $A$ and $B: 2$ months of age, $C$ and $D: 3$ months of age, $E$ and $F: 4$ months of age. ( $A$ and $B$ ) Enhancing mass-like lesion in the tectal area causing aqueductal stenosis and hydrocephalus. Diffuse high signal T2 intensity in the cerebral white matter was noted. (C-E) Serial scans revealed progressive cortical atrophy and extension of cerebral white matter T2 high signal intensity and a tectal mass-like enhancing lesion. Arrow; tumor-like lesion in the tectal area. 
Head circumference measured at 5 months was $46 \mathrm{~cm}$ (> 97 percentile). After re-reviewing the clinical features and brain MRI findings, Alexander disease was suggested as a possible diagnosis. Subsequent direct sequencing of GFAP confirmed a de novo missense mutation (c.1096T>C, Y366H; Fig. 2), which has been reported previously. ${ }^{5}$ At his last follow-up visit at 28 months, psychomotor development was severely retarded; the patient could neither control his head, nor fix and follow an object. He was completely bedridden, fed via a gastric tube, and dependent on a home ventilator.

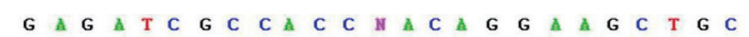

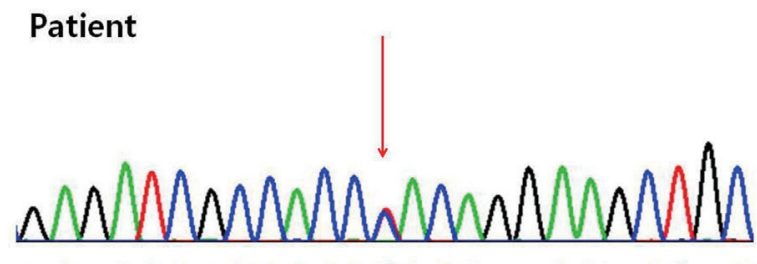

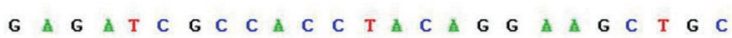

Father

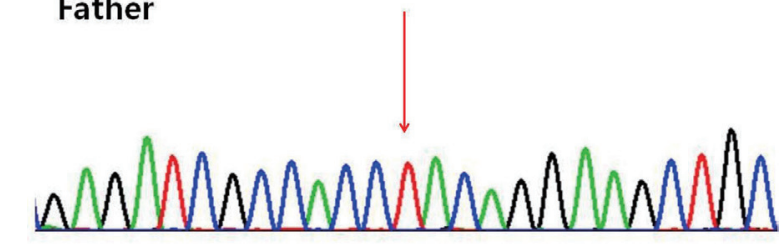

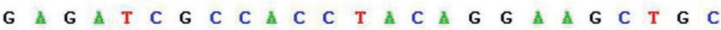

Mother

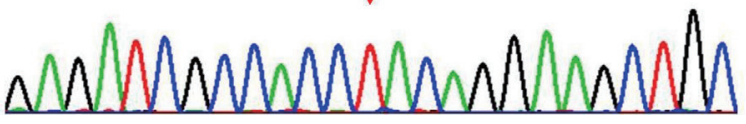

Fig. 2. A de novo missense mutation of c.1096T $>C$ (Y366H) was confirmed by GFAP direct sequencing.

\section{Discussion}

Alexander disease is classically described as a leukoencephalopathy with macrocephaly; however, considerable variation exists in the clinical manifestation of this rare disease. While infantile or early onset Alexander disease is characterized by progressive psychomotor regression, ataxia, and prominent upper motor neuron signs, the neonatal form has somewhat different clinical features as reported in a limited number of patient cases. Springer et al. reported 3 cases of Alexander disease with neonatal onset, ${ }^{4)}$ summarized as having the following differentiating clinical features: intractable seizures, rapid progression, hydrocephalus with intracranial pressure primarily caused by aqueductal stenosis, as well as absence of hyperreflexia and spasticity. Although the onset of the case discussed here was slightly delayed until after the neonatal period (50 days old), the overall clinical features of this case were more consistent with the neonatal form of Alexander disease. Specifically, obstructive hydrocephalus with a tectal mass-like enhancing lesion was consistent with the diagnosis of a brain tumor, which would be unusual for this age but still included in the differential diagnosis. These atypical clinical features delayed the ultimate diagnosis of Alexander disease, which was suspected only after the progression of white matter signals was identified on serial brain MRIs. Subsequently, we confirmed the diagnosis of Alexander disease by GFAP sequencing.

A recent comprehensive genotype-phenotype correlation study found that half of all mutations found in Alexander disease affected 1 of 4 amino acids in the GFAP peptide residue (R79, R88, R239, and R416). ${ }^{2)}$ They also reported that the patients with R79 and R239 mutations tended to have seizures, macrocephaly, and classic radiologic features at an early age of onset. However,

Table 1. Clinical Features of the 3 Reported Alexander Disease Patients with Mutation at GFAP Y336 Peptide Residue

\begin{tabular}{|c|c|c|c|}
\hline & The present case & Case $1^{5)}$ & Case $2^{6)}$ \\
\hline cDNA change & c.1096T>C & C.1096T>C & c. $1097 A>G$ \\
\hline Aminoacid change & Y366H & Y366H & Y366C \\
\hline Sex & Male & Male & Female \\
\hline Age of onset (months) & 2 (7 weeks) & 1 & 4 \\
\hline Seizure & + & + & + \\
\hline Macrocephaly/hydrocephalus & $+/+$ & $-/ N A$ & $+/+$ \\
\hline Burbar and or pseudobulbar sign & - & NA & NA \\
\hline Ataxia & - & NA & + \\
\hline Cognitive dysfunction & + & + & + \\
\hline Hyperreflexia & - & NA & + \\
\hline
\end{tabular}

${ }^{\star} \mathrm{NA}$, not available 
there have been only few reported cases with mutations involving Y366 residue as the present case. From the literature review, we found two patients with the same GFAP peptide residue mutiation. ${ }^{5,6}$ Including the present case, we summarized the clinical features of two other patients with Y366 mutation found in other literatures in Table 1. Although the sample size was small, patients with Y366 mutation tended to present early onset symptoms with clinical features favoring the neonatal form such as intractable seizures and hydrocephalus.

Only two genetically confirmed Alexander disease cases have been reported in Korea (R239L and K86E) to date. ${ }^{7.8}$ All two cases had infantile onset (8 months and 10 months) and revealed classical symptoms and signs such as developmental delay, macrocephaly, and seizures. The present case highlights a rare but distinct clinical presentation of neonatal form of Alexander disease. Since intractable seizures and obstructive hydrocephalus with mass-like brain stem lesions are predominant features of the neonatal form of Alexander disease, heightened awareness of these features may lead to a timely clinical diagnosis. Moreover, the possible clinical correlation between Y366 residue mutations and neonatal Alexander disease needs further investigation in future studies.

\section{Acknowledgement}

This research was supported by Basic Science Research Program through the National Research Foundation of Korea(NRF) funded by the Ministry of Science, ICT \& Future Planning (2013R1A1A1008888)

\section{References}

1. Alexander WS. Progressive fibrinoid degeneration of fibrillary astrocytes associated with mental retardation in a hydrocephalic infant. Brain $1949 ; 72: 373-81$.

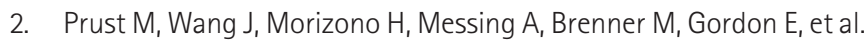
GFAP mutations, age at onset, and clinical subtypes in Alexander disease. Neurology 2011;77:1287-94.

3. Singh N, Bixby C, Etienne D, Tubbs RS, Loukas M. Alexander's disease: reassessment of a neonatal form. Childs Nerv Syst 2012;28:2029-31.

4. Springer S, Erlewein R, Naegele T, Becker I, Auer D, Grodd W, et al. Alexander disease--classification revisited and isolation of a neonatal form. Neuropediatrics 2000;31:86-92.

5. Li R, Johnson AB, Salomons G, Goldman JE, Naidu S, Quinlan R, et al. Glia fibrillary acidic protein mutations in infantile, juvenile, and adult forms of Alexander disease. Ann Neurol 2005;57:310-26.

6. Hartmann H, Herchenbach J, Stephani U, Ledaal P, Donnerstag F, Lucke T, et al. Novel mutations in exon 6 of the GFAP gene affect a highly conserved if motif in the rod domain $2 \mathrm{~B}$ and are associated with early onset infantile Alexander disease. Neuropediatrics 2007;38:143-7.

7. Park GM, Ko JH, Min KS. A case of infantile Alexander disease. J Korean Child Neurol Soc 2009;17:215-20.

8. Lee JM, Kim AS, Lee SJ, Cho SM, Lee DS, Choi SM, et al. A case of infantile Alexander disease accompanied by infantile spasms diagnosed by DNA analysis J Korean Med Sci 2006; 21:954-7. 